\title{
BERND MARTIN
}

\section{Aussenhandel und Aussenpolitik Englands unter Cromwell}




\section{AUSSENHANDEL UND AUSSENPOLITIK ENGLANDS UNTER CROMWELL VON

\author{
BERND MARTIN
}

WENN die Entstehung des europäischen Staatensystems nach dem Ende der Religionskriege nicht allein unter dem Primat der Außenpolitik als "Vorgang äußerer Staatenbildung ${ }^{\iota}$ ) verstanden werden kann, sondern stärker unter dem Gesichtspunkt einer von gesellschaftlichen Interessen bestimmten Kongruenz innerer und auswärtiger Politik zu sehen ist, so gilt die Nationwerdung Englands im Rahmen des neuzeitlichen Mächteverbandes geradezu als Modellfall. Dieser in dem englischen Bürgerkrieg und der sich anschließenden Herrschaft Cromwells kulminierende ProzeB wurde daher von Sozialwissenschaftlern gern als exemplarischer Beweis zur Untermauerung ibrer Theorien herangezogen. So datiert Marx mit der englischen Navigationsakte den Beginn des Kampfes zwischen bürgerlichfreien Konkurrenzgesellschaften um die Aufteilung der Weltmärkte2); die Außenpolitik Cromwells habe daher -- nach Kuczynski

1) Otto Hintze: Staatenbildung und Verfassungsentwiddung. Eine historisch-politische Studie. In: Ders. Staat und Verfassung. Gesammelte $\mathbf{A b}$ handlungen zur allgemeinen Verfassungsgeschichte. Göttingen 81962. S. 34. 7) Karl Marx, Friedrich Engels: Werke. Hrsg. vom Institut für Marxismus-Leninismus beim ZK der SED. 38 Bde. Berlin (Ost) 1956 ff. (MEW Bd. 3 S. 58 ff.: Karl Marx, Friedrich Engels: Die deutsche Ideologie). Hingegen fehlt bei Marx eine genaue Analyse der ökonomischen Voraussetzungen wie der sozialen Auswirkungen der englischen Revolution. Im ersten Band des Kapitals findet sich in einer Fußnote lediglich die Bemerkung: „Solange die Republik währte, erhob sich die englische Volksmasse in allen Schichten aus der Degradation, wozu sie unter den Tudors gesunken war" (MEW Bd. 23 S. 776). Ebenfalls unzutreffend ist seine Erklärung der Restauration: "In der Tat, die englische Republik unter Cromwell scheiterte an - Irland" (MEW Bd. 32 S. 638 Brief vom 29. Nov. 1869 an Kugelmann). Für eine marxistische Interpretation dieser „bürgerlichen Revolution " siehe Jürgen Kuczynski: Darstellung der Lage der Arbeiter in England 1640-1760. Berlin (Ost) 1964. S. $153 \mathrm{ff}$., der jedoch weder Marx' Behauptung, in England habe eine kapitalistische Gesell- 
- den Belangen der herrschenden städtischen Bourgeoisie entsprochen ${ }^{3}$ ). Obgleich Hallgarten geographische und historische Besonderheiten gelten läßt, konstatiert er ebenfalls eine - allerdings von der "halbproletarischen Armee“ getragene - Expansionspolitik, die mit Ubernahme des holländischen Weltfrachtmonopols einen Grundstein zur imperialistischen englischen Ausdehnung gelegt habe4). Demgegenüber betont die religionssoziologische Deutung Max Webers die Einwirkung des asketisch-puritanischen Geistes auf die sozialpolitischen Anschauungen und versteht daher Kriege lediglich als zweckrationale Auscinandersetzung, als Belebung für den Kapitalismus ${ }^{5}$ ). In Weiterführung des Weberschen Ansatzes hat die von Tawney begründete sozialgeschichtliche Schule ${ }^{6}$ ) die gesellschaftlichen Wurzeln des alle Bereiche umfassenden Umwälzungsprozesses im England des 16. und 17. Jahrhunderts untersucht und dabei die Wechselwirkung zwischen religiöser Erneuerung und sozialem Wandel aufgezeigt. Die besitzorienticrte Lebensweise, der säkularisierte orthodoxe Calvinismus, habe erstmals reine Handelskriege ermöglicht, wie die gegen Holland. In ihnen sei der Staat als gemeinsam von den Bürgern zu schuitzendes Eigentum verteidigt worden. Diesen entscheidenden Einfluß der kapitalkräftigen Oberschicht auf die englische Politik hatten bereits zeitgenössische politische Theoretiker, Hobbes, Milton, Harrington und Radikaldemokraten wie Lilburne und Winstanley?), erkannt, jedoch unterschiedliche Konsequenzen

schaft bestanden, noch die Hills (s. u. Anm. 11) von einer Eigentumsrevolution gelten läßst.

3) Kuczynski S. 168

4) George F. W. Hallgarten: Imperialismus vor 1914. Die soziologischen Grundlagen der Außenpolitik europäischer Großmächte vor dem Ersten Weltkrieg. 2 Bde. München '1963. Bd. 1 S. 42 f.

7) Max Weber: Gesammelte Aufsätze zur Religionssoziologie. Tübingen 41947, insbesondere: „Die protestantische Ethik und der Geist dcs Kapitalismus", bzw. Bd. I S. 394.

o) R. H. Tawney: Religion and the Rise of Capitalism. New York 1926 (dt:: Religion und Frühkapitalismus, Bern 1946). Zu den fundiertesten sozialgeschichtlichen Darstellungen der Tawney-Schule gehören: Margaret James: Social Problems and Policy during the Puritan Revolution 16401660. London 1930 (Reprint 1966) sowie - über Tawney hinausgehend Lawrence Stone: The Crisis of the Aristocracy 1558-1641. Oxford 1965.

T) Dazu allgemein Perez Zagorin: A History of Political Thought in the English Revolution. London 21965 und Eric Voegelin (Hrsg.): Zwischen Revolution und Restauration. Politisches Denken in England im 17. Jahrhundert. München 1968. Speziell zu Hobbes: Bernard Willms: Die Antwort des Leviathan. Thomas Hobbes' politische Theorie. Neuwied-Ber- 
aus dieser Einsicht gezogen. Im Gegensatz zu dieser zeitgebundenen wie zu der sozialhistorischen modernen Betrachtung erweisen sich die traditionelle Deutung der "Whig-Historians"8) mit ihrer Glorifizierung Cromwells als Gründer des britischen Weltreiches und die konservativ abwertenden Interpretationen ${ }^{\circ}$ ) dieser als "Interregnum “ oder „Great Rebellion“ bezeichneten Epoche als unbefriedigend einseitige Erklärungsversuche einer der wichtigsten Zeitabschnitte der englischen Geschichte.

Angesichts der breit gefächerten und dabei höchst kontroversen Interpretationen verwundert es, $\mathrm{daB}$ weder die individualisierendpersonalistische Geschichtsbetrachtung noch die sozialhistorische, ja nicht einmal die marxistische ihre Ergebnisse und die daraus abgeleiteten Theorien an einer zentralen Fragestellung überprüft hat, nämlich der von den Auswirkungen einer inneren Umgestaltung auf den Sektor der auswärtigen Beziehungen ${ }^{10}$ ). Wenn die Erkenntnis als

lin 1970. Zu Milton: Don M. Wolfe: Milton in the Puritan Revolution. London 1941, Reprint 1963; zu Harrington: Charles Blitzer: An Immortal Commonwealth. The Political Thought of James Harrington. Yale Univ. Press 1960; zu Lilburne und Winstanlcy: P. Gregg: Free-born John, A Biography of John Lilburne. London 1961, Lewis H. Berens: The Digger Movement in the Days of the Commonwealth as Revealed in the Writings of Gerrard Winstanley ... London 1906 und Eduard Bernstein: Sozialismus und Demokratie in der großen engliscten Revolution. Stuttgart-Berlin 41922.

8) Obwohl ihnen das Verdienst zukommt, in Standardwerken Cromwell und seine Zeit erstmals wissenschaftlich aufgearbeitet zu haben, interpretieren Samuel Rawson Gardiner (History of the Commonwealth and Protectorate 1649-1660. 3 Vls. London 1894 ff.) und Charles Firth (Oliver Cromwell and the Rule of the Puritans in England. London 1900) diesen Zeitabschnitt befangen im liberalen Wirtschafts- und Empiredenken Englands um die Jahrhundertwende.

D) Als moderner Exponent dieser Richtung kann Hugh R. Trevor-Roper (Religion, the Reformation and Social Change. London 1967. Dt.: Religion, Reformation und sozialer Umbruch. Die Krise des 17. Jahrhunderts. Frankfurt am Main 1970) gelten.

10) Weder über den Außenhandel noch über die auswärtige Politik des Commonwealth bzw. Protektorats existieren Monographien. Der Aufsatz von Menna Prestwich (Diplomacy and Trade in the Protectorate. In: „Journal of Modern History“, 1950, S. 103-121) bietet einen Uberblick, in dem jedoch die funktionale Abhängigkeit der AuBenpolitik von der inneren Entwicklung zu wenig berücksichtigt und überdies Cromwell als religiös verblendeter außenpolitischer Ignorant ïberzeichnet wird. Allgemein zum Außenhandel: $G$. D. Ramsay: English Overseas Trade during the Centuries of Emergence. London 1957. 
gesichert vorausgesetzt werden darf, daß wirtschaftliche Kräfte als ein entscheidendes Movens bei der Umschichtung der englischen Sozialstruktur seit Heinrich VII. anzusehen sind und in England wie in keinem anderen Staat dic Entstehung einer weitgehend an einem geschlossenen Binnenmarkt orientierten Gesellschaft, einer „marketplace society“, begünstigten ${ }^{11}$ ), dann vermochte diese in ihrem Selbstverständnis sich frühzeitig als homogen begreifende und als Folge der verweltlichten Prädestinationslehre12) sich als auserwählt-überlegen fühlende Nation die internationalc Bühne schwcrlich auf einem anderen Wege als über den Außenhandcl zu beschreiten. Nicht dynastische Bindungen noch auch religiöse Fragen als vielmehr der Außenhandel standen im 17. Jahrhundert im Mittelpunkt des außenpolitischen Interesses ${ }^{13}$ ) der herrschenden, d. h. wahlberechtigten, zahlenmäBig kleinen Oberschicht ${ }^{14}$ ). Im folgenden soll daher für die Regierungsjahre Cromwells, das Dezennium 1649 bis 165815), untersucht werden, wie sich die vom Bürgerkricg erschüttcrte, dabei aber endgültig ihrer fcudal-frühabsolutistischen Fesseln ledig ge-

11) Dazu Christopher Hill: Reformation to Industrial Revolution. A Social and Economic History of Britain 1530-1780. London 1967.

12) Obwohl das Parlament den Arminianismus während des Bürgerkrieges zugunsten des orthodoxen Calvinismus verwarf (Solemn League and Covenant 1643, Westminster Confession 1647), um die Massen in der presbyterianisch-calvinistischen Kirchenzucht zu halten und die von Sekten ausgehende politische und soziale Gefahr zu bannen, bekannte sich Milton bereits zur verweltlichten Prädestination (Wolfe S. $23 \mathrm{ff}$.), bevor independentisches Toleranzdenken nach 1649 dic orthodoxe Auffassung auch im Parlament zuriickdrängte.

19) Ernst Schulin: Handelsstaat England. Das politische Interesse der Nation am Außenhandel vom 16. bis ins frühe 18. Jahrhundert. Wiesbaden 1969.

14) Etwa 10\% der männlichen erwachsenen Bevölkerung, ca. 110000 Pcrsonen bei einer Gesamtbevölkerung von ca. 5,2 Millionen in England, waren wahlberechtigt (Voegelin S. $106 \mathrm{ff}$. und Christopher Hill: The Century of Revolution 1603-1714. London 1961. S. $43 \mathrm{ff}$.) Das an Landbesitz im Wert von $40 \mathrm{~s}$, in den Städten an die Zugehörigkeit zu einer Korporation gebundene Zensuswahlrecht wurde 1653 zugunsten des Nachweises von 200 Pfund Vermögen abgewandelt. Damit schaltete die wohlhabende Gentry die politisch unzuverlässigen Klassen der selbständigen Kleinbauern und der Handwerker von der Politik aus (Hill, Century of Revolution S. 135).

15) Obwohl Cromwell erst im Dezember 1653 als Lordprotektor nominel] die Regierung übernahm, war er nach der Hinrichtung Karls I. (30. 1. 1649) die beherrschende Figur in dem jährlich neu gewählten Staatsrat während der Zeit des Commonwealth. Cromwell starb am 3. September 1658. 
wordene16) englische wirtschaftsorientierte Gesellschaft außenpolitisch artikulierte. An den drei wichtigsten auswärtigen Problemkreisen, dem englischen Verhältnis zu Holland, den Beziehungen zu Spanien und der englischen Ostseepolitik, gilt es aufzuzeigen, welche politischen Kräfte mit welchen Motiven auf die Entscheidungen bestimmend einwirkten oder vergebens mit einer sich immer stärker verselbständigenden staatlichen Führungsspitze um den Kurs der AuBenpolitik rangen. Unter weitgehender Ausklammerung von Fakten wird dabei auch aufzuhellen sein, welche damaligen Maßnahmen außenwirtschaftlich-strategische Leitlinien der britischen Politik bis in unsere Zeit determinierten.

Die Vcreinigten Niederlande, die ihre staatliche Unabhängigkeit weitgehend der protestantisch-elisabethanischen Protektion zu verdanken hatten ${ }^{17}$ ), waren wie die Insel England aufgrund ihrer geographisch geschuitzten Lage am Rande Westmitteleuropas beide bestens geeignet, sich des vornchmlich über See abgewickelten Warenaustausches zwischen den ibcrischen Kolonialmächten und Nord- bzw. Mittcleuropa als Zwischenhändler zu bemächtigen. Fehlende ideologisch-religiöse Spannungen und seit der Ausrufung des englischen Commonwealth ein nahczu verwandtes Gesellschaftssystem boten scheinbar ideale Voraussetzungen für ein gutnachbarschaftliches Verhältnis. Doch gcrade dic natürliche Gleichartigkeit und die Konvergenz der Systeme schürten Animositäten, die auf englischer Seite wegen der wirtschaftlichen Uberlegenheit des als übermächtig empfundenen Konkurrenten ${ }^{18}$ ) stärker zutage traten als in Holland, das seine nach dem englischen Bürgerkrieg gefestigte Vormachtstellung bewahren wollte. Obwohl holländische Schiffe kaum

10) Abschaffung der königlichen prärogativen Gerichtshöfe (Star Chamber, Council of the North) und der Vormundschaft der Krone bei minderjährigen Erben (Wardship). Siehe dazu die von Samuel $R$. Gardiner herausgegebenen "The Constitutional Documents of the Puritan Revolution $1628-1660^{\prime c}$. London ${ }^{31906 .}$

17) Unterstützung des niederländischen Freibeitskampfes durch den englischen Seesieg über die Armada 1588 im bis 1604 andauernden Krieg mit Spanien.

18) Zur Organisation der Volkswirtschaft in den Niederlanden: Etienne Laspeyres: Geschichte der volkswirtschaftlichen Anschauungen der Niederländer und ihre Literatur zur Zeit der Republik. Leipzig 1863. Reprint Niewkoop 1951. Für den englisch-holländischen Dualismus: Charles Wilson: Profit and Power. A Study of England and the Dutch Wars. London - New York - Toronto 1957. 
englische Häfen anliefen $\left.{ }^{18}\right)$, monopolisierten die Niederlande mit ihrer der englischen etwa siebenfach überlegenen Tonnage den lukrativen Nord-Süd-Frachtverkehr und auch zusehends den Kolonialhandel $\left.{ }^{20}\right)$. Demgegenüber wies der vorwiegend über London abgewickelte englische Außenhandel seit etwa 1616 einen starken Importüberhang auf und bewirkte damit eine wirtschaftliche Dauerkri$\mathrm{se}^{21}$ ). Der englische Export, zu etwa $3 /$, Tuche ${ }^{22}$ ), war infolge verfehlter dirigistischer Eingriffe der frühen Stuarts's) und mit dem Verlust des mitteleuropäischen Marktes während des Dreißigjährigen Krieges stetig zurückgegangen ${ }^{24}$ ). In den Zentren der Tuchindustrie, in den gewerbetreibenden Städten und besonders in London wurde daher ein verarmtes Proletariat zusammen mit einem in seiner Existenz ebenfalls durch die Depression bedrohten unteren Mittelstand, dem Kleinhandwerk, für revolutionär-chiliastische Bewegungen anfällig und stellte eine stete Gefahr für die herrschende soziale Ordnung dar ${ }^{25}$ ).

19) Bereits um 1640 war die holländische Schiffahrt aus dem direkten Englandverkehr verdrängt worden. Dazu: $O$. A. Johnsen: The Navigation Act of 9 October 1651. In: „History“, 1949, S. 89-96.

20) Zur zahlenmäBigen und technischen Uberlegenheit der holländischen Handelsflotte, die stärker war als die der übrigen europäischen scefahrenden Nationen zusammen: Wilson S. $41 \mathrm{ff}$, und Ludwig Bentin: Die britische Navigationsakte von 1651. In: „Die Welt als Geschichte", 1952, S. 44-53.

21) B. E. Supple: Commercial Crisis and Change in England 1600-1642. A Study in the Instability of a Mercantile Economy. Cambridge 1959. Nach einer zeitgenössischen mit unzulänglichen Mitteln erstellten Handelsbilanz bestand in den Jahren 1613 und 1614 zusammen ein Exportüberhang von über einer halben Million Pfund, während nach einer exakteren Außenhandelsbilanz des „Privy Council“ für 1616 die Einfuhren die Exporte um das Vierfache übertrafen. Abdruck beider Bilanzen bei $J$. P. Cooper und J. Thirsk (Hrsg.): Seventeenth-Century Economic Documents. Oxford 1972. S. 454 ff. und 459 ff.

22) Supple S. 6 und Peter J. Bowden: The Wool Trade in Tudor and Stuart England. London 1962.

23) Zum sog. „Cockayne Projekt", der Ubernahme des Tuchhandels von der Handelskompanie der „Merchant Adventurers" durch ein königliches AuBenhandelsmonopol 1614-1617, siehe Supple S. 33 ff.

24) Ebenda S. 52 ff. und exemplarisch für den Ostseehandel: $R$. W. K. Hinton: The Eastland Trade and the Common Weal in the Seventeenth Century. Cambridge 1959.

25) Lebten zu normalen Zeiten 8\% der Bevölkerung unter dem Existenzminimum, so waren es in den 40er Jahren des 17. Jahrhunderts infolge des Bürgerkrieges ca. 20\% (M. Ashley: England in the Seventeenth Century. Baltimore 1952. S. 24). Zu dem VerelendungsprozeB der Lohnabhängigen 
Unter dem wachsenden Druck der sich über die siegreiche puritanische Revolutionsarmee Gehör verschaffenden unteren Mittelschichten ${ }^{26}$ ) wurde die Commonwealth-Regierung sogleich nach ihrer Konstituierung gezwungen, wirtschaftliche Fragen vordringlich zu behandeln. Die Säuberung des Parlaments von Angehörigen des presbyterianischen Establishments, der "landed gentry ${ }^{\text {" }}$ und des alteingesessenen Londoner Kaufmannstandes, durch das puritanische Heer verschaffte der neuen in der Auseinandersetzung mit dem König entstandenen „großbürgerlichen“, im Religiösen meist independentischen Klasse der "trading gentry “ und des freien Großkaufmanns, dem ,interloper“, dic geeignete Plattform, um ihre außenwirtschaftlichen Interessen zu vertreten ${ }^{27}$ ). Mit einigem Recht

siehe allgemein Karl Marx: Kapital, Bd. 1, Kap. 24, Kuczynski S. $130 \mathrm{ff}$. und Hill S. 14 ff.; speziell für die Tuchindustrie Supple. Die radikaldemokratischen Führer entstammten in der Regel dem von der Wirtschaftskrise schwer getroffenen Londoner mittleren und klcineren Kaufmannstand. So absolvierte John Lilburne ab 1630 eine siebenjährige Lehrzeit bei einem Londoner Leinwandhändler. Gerrard Winstanley ging 1643 als Tuchhändler bankrott, während sein Mitstreiter William Walwyn als Mitglied der „Merchant Adventurers" zu den wohlhabenden Tuchhändlern zähite.

20) Zur Agitation der Levellers in der Armee 1647-1649 und ihren politischen und wirtschaftlichen Programmen siehe die Dokumente bei $J$. $P$. Kenyon: The Stuart Constitution 1603-1688. Documents and Commentary. Cambridge 1966. S. 302 ff. Ferner $H$. N. Brailsford: The Levellers and the English Revolution. Stanford Univ. Press 1961 und Georg Lenz: Demokratie und Diktatur in der englischen Revolution 1640-1660. München/ Berlin 1933. Beiheft 28 der Historischen Zeitschrift.

27) Die Trennungslinien zwischen beiden Lagern sind fließend und keinesfalls allein von wirtschaftlichen Interessen bedingt. Gerade bei den Angehörigen der Gentry wurde der politische Horizont in viel stärkerem Maße von weltanschaulichen Auffassungen und familiär-regionalen Bindungen geprägt als durch materiellen Wohlstand. Im Gegensatz zur Forschung der 1950er Jahre, die schematisierende Klasseneinteilungen verwarf (z. B. die Kontroverse um die Gentry zwischen $R$. $H$. Tawney: The Rise of the Gentry 1558-1640. In: „The Economic History Review“, 1941, S. 1-38 und die ebendort (Supplements 1, 1953) zu findende Erwiderung von $\boldsymbol{H}$. $R$. Trevor-Roper; ferner die wahlsoziologische Untersuchung von $D$. Brunton und $D$. H. Pennington: Members of the Long Parliament. Aberdeen 1954), werden in jüngeren sozialgeschichtlichen Arbeiten stärker wirtschaftliche Interessengruppierungen analysiert. So für die Säuberung des Parlaments am 6. Dez. 1648 David Underdown: Pride's Purge. Politics in the Puritan Revolution. Oxford 1971 und für die Kaufleute: Robert Brenner: The Civil War Politics of London's Merchant Community. In: „Past and Present", No. 58, Febr. 1973, S. 53-107. 
wurde daher das Rumpfparlament bereits von den Zeitgenossen als "trading Parliament" bezeichnet. Die staatliche Exekutive, der „Council of State", war, obgleich seine Zusammensetzung eher das konservativ ständische Element hoher Militärs und einflußreicher Landbesitzer widerspiegelteze), sowohl von der Armee als Garant der revolutionären Ordnung als auch von der Londoner City, dem Hauptgeldgeber, abhängig. Administrative Maßnahmen, wic die Gründung eines Volkswirtschaftsrats29) und das Handelsverbot mit den royalistischen Kolonien in Amerika ${ }^{30}$ ), befriedigten dann auch Hauptanliegen der auf weltweiten, freien Handel drängenden independentischen Kreise, während die forcierte Aufrüstung der $\mathrm{Ma}$ rines1) die Wirtschaft belebte und überdies auch den Wünschen der traditionellen, in Handelskompanien organisierten Kaufleute cntsprach. Die Förderung der Marine durch kapitalkräftige Handelskreise wurde im wesentlichen von drei Uberlegungen bestimmt: 1. die von einem stehenden Heer ausgehendc soziale und politische Bedrohung zu kontern ${ }^{32}$ ), 2. profitable, hochspezialisierte und von Importen abhängige Industriezweige auszubauen ${ }^{33)}$ und 3 . die Marine zum Schutz der Seeverbindungen einzusetzen und zur Aus-

${ }^{28}$ ) In dem Gesetz zur Konstituierung eines 41köpfigen Staatsrates wird die Förderung des Handels als programmatischer Punkt angeführt (C. H. Firth und $R$. S. Rait, Hrsg.: Acts and Ordinances of the Interregnum 1642-1660. 3 Vols. London 1911. II S. 2 ff.) Zur Zusammensetzung: Gardiner, History of the Commonwealth Bd. I S. 6 und M. Ashley: Financial and Commercial Policy under the Cromwcllian Protectorate. Oxford 21962. S. 6.

29) Firth/Rait II S. 403 ff. Der am 1. August 1650 begrïndete „Council of Trade" hatte nur beratende Funktionen. In diesem etwa zur Hälfte mit Kaufleuten besetzten Rat waren die "Interlopers" tonangebend (Brenner S. 102).

30) Firth/Rait II S. 425 ff: Parlamentsbeschluß vom 3. Okt. 1650. Für den EinfluB der "Interlopers" siehe Brenner S. 103.

31) Zu der unter „Marinestaatssekretär ${ }^{\text {st }}$ Sir Henry Vane the Younger betriebenen Aufrüstung siehe Hinton S. 96 und die Biographie von Violet A.Rowe: Sir Henry Vane the Younger. A Study in Political and Administrative History. London 1970.

s2) Der allein von der Marine geführte „Handelskrieg“ gegen Holland war daher in der revolutionären Armee unpopulär, zumal im Parlament sogleich wieder Stimmen für eine Reduzierung des Heeres laut wurden (Wilbur Cortez Abbott: The Writings and Speeches of Oliver Cromwell. 4 Vols. Harvard Univ. Press 1937 ff. Vol. II S. 556 am 2. Juni 1652) und der Sold für die Matrosen im Dez. 1652 auf Kosten der Armee beträchtlich angehoben wurde (Ebenda S. 606 ff.). Dazu auch Wilson S. $61 \mathrm{ff}$.

38) Die Werften waren von Schiffsbaumaterialien des Ostseeraumes, Masten, Segeltuche, Eisenwaren, abhängig. Siehe die Tabellen bei Hinton S. 38 f. 
bildung des Nachwuchses für die Handelsschiffahrt, gewissermaßen als "Schule der Nation“, zu benutzen. Diese Interessenkoalition sollte für die innen- wie außenpolitische Entwicklung Englands richtungweisend sein und die im Industriezeitalter noch stärker technologisch-wirtschaftlich bedingte Zusammenarbeit von Schwerindustrie und Marine bei den Seemächten antizjpieren ${ }^{34}$ ).

Handelspolitische Motive, auf seiten der ,interlopers“" wie der Handelskompanien, machtpolitische Überlegungen, nämlich einc über See vorgetragene Konterrevolution zu vereiteln ${ }^{35}$ ), und das Moment der sozialen Integration gaben dann auch den Ausschlag, die bestehenden, aber meist übergangenen Schiffahrtsgesetze in der Navigationsaktesv) für alle Importe und zur Protcktion der heimischen Fischfangindustrie verbindlich zu kodifizieren. Dieses Gesetz diente daher in der Situation des Jahres 1651, nach gescheiterter Invasion des jungen in Holland residierenden Königs im Bündnis mit den Schotten ${ }^{37}$ ), einer endgültigen politischen Konsolidierung der neuen Regierung; außenpolitische Verwicklungen waren damit ursprünglich nicht erwartet worden, mußten sich aber zwangsläufig bei einem Wiedererstarken des englischen Scepotentials mit der holländischen Konkurrenz ergeben.

Nicht über die gewaltsame Durchsetzung der Navigationsakte, sondern aus einer maritimen Strategie Englands, in der die Niederlande als britische Gegenküste und die dazwischenliegenden Gewässer, der „English Channel“, als englisches Hoheitsgebiet angesehen wurden, weiteten sich Zwischenfälle, wie gewaltsame Durchsuchungen holländischer Schiffe oder die Nichtbcachtung der englischen Schiffen gegenüber gebotenen Grußpflicht ${ }^{38}$ ), zu einem un-

s4) So z. B. in den USA und Japan.

35) Dieser Gesichtspunkt besonders stark bei Bentin.

sin) Neben den Darstellungen von Bentin, Johnsen das in manchen Partien überholte Standardwerk Lawrence A. Harper: The English Navigation Laws. A Seventeenth Century Experiment in Social Engineering. New York 1939 und den Aufsatz von J. E. Farnell: The Navigation Act of 1651, the First Dutch War, and the London Merchant Community. In: „The Economic History Review", Vol. XVI, 1963/4, S. 439-454. Text der Akte vom 9. Okt. 1651 bei Firth/Rait II S. 559 ff. und Cooper/Thirsk S. $502 \mathrm{ff}$.

s7) Niederlage der mit dem König verbündeten Schotten bei Worcester am 3. Sept. 1651 gegen das von Cromwell geführte Heer.

38) Wilson S. $57 \mathrm{ff}$. Seitdem Frankreich 1648 den Import englischen Tuches gesperrt hatte und die Commonwealth Regierung mit einem Importembargo auf französische Waren (Firth/Rait II S. $239 \mathrm{f}$. vom 
erklärten Seekrieg aus. Das von Grotius verfochtene Prinzip des „mare librum" stand dem englischen vom „mare clausum" diametral entgegen ${ }^{3 y}$ ). Die Einzigartigkeit des englischen Seekriegsrechts, wie die Prisenordnung, Bestimmungen über Konterbande und schließlich sogar die Legitimität einer über den Gegner zu verhängenden Hungerblockade'0), wurde erstmals im Krieg gegen den holländischen Rivalen konsequent praktiziert und für die Zukunft präjudiziert.

Gegen den von allen Schichten, besonders vehement von den radikalen Sekten propagierten antiholländischen nationalen Volkskrieg ${ }^{41}$ ) erhoben sich nur wenige Stimmen, die, angeführt von Cromwell, vorgaben, man könne um der gemeinsamen protestantischen Sache willen nicht über den Nachbarn herfallen ${ }^{42}$ ). Ausschlaggebend für den endgültigen englischen Kriegsentschluß wurden das Scheitern des Cromwellschen Projektes, die Gegenküste - das spanische Dünkirchen - mit diplomatischen Mitteln zu beherrschen ${ }^{43}$ ), und - wohl

28. August 1649) geantwortet hatte, herrschte zwischen Frankreich und England bis 1657 ein unerklärter Handelskrieg zur See, in dem die Engländer willkürlich holländische Schiffe nach Konterbande durchsuchten.

s9) Hugo Grotius: Mare Librum (1613) forderte die noch durch Karl I. angeregte Gegenschrift von John Selden: Mare clausum seu Dominium Maris (1632) heraus. Die Grenzen der englischen Hoheitsgewässer wurden jedoch von Selden nicht exakt beschrieben.

40) Die von den Holländern verfochtene Rechtsauffassung "free ship free good“ und die enge Begrenzung der Konterbande auf Munition lehnten die Engländer ab. Statt dessen wurden von englischer Seite sogar Getreideladungen auf neutralen Schiffen als Konterbande angesehen und konfisziert. Lediglich nach dem zweiten holländischen Seekrieg beugten sich die Engländer 1667 vorübergehend den holländischen Auffassungen. In allen drei Kriegen mit Holland blockierte die „Royal Navy“ die holländische Küste und unterband damit die für die Versorgung der Bevölkerung notwendigen Getreidelieferungen. Dazu Wilson S. 61 ff. und S. 141 sowie $M$. Ashley, Financial and Commercial Policy S. 170.

11) So vertrat Lilburne eine englische Handelssuprematie über Holland (Zagorin S. 18), befürwortete Winstanley im 1652 publizierten „Law of Freedom" - wie die von ihm bekämpften Kaufleute - die Navigationsakte (Berens S. 248) und forderten die radikalen „5th Monarchy Men" die Unterwerfung Hollands unter die Herrschaft Christi, d.h. der ,godly English" (P. G. Rogers: The Fifth Monarchy Men. Oxford 1966. S. 146).

12) Gardiner, History of the Commonwealth and Protectorate Vol. II S. 111 und Christopher Hill: God's Englishman. Oliver Cromwell and the English Revolution. London 1970 S. 131.

43) Gardiner, History of the Commonwealth and Protectorate Vol. II S. 103 ff.: Cromwells Plan, durch Besetzung des von den Spaniern belagerten französischen Dünkirchens Holland wirtschaftlich und strategisch in 
noch entscheidender - der desolate Zustand der Staatsfinanzen. Allein die Deckungslücke für den Marinehaushalt betrug 1652 annähernd eine halbe Million Pfund, zu der ebenfalls nicht gedeckte Forderungen des Heeres und Soziallasten als Folge der vorausgegangenen Kriege traten $\left.{ }^{44}\right)$. Eine Erhöhung der von den Besitzenden getragenen Vermögenssteuer ${ }^{45}$ ), die Haupteinnahmequelle des Staates, war nicht denkbar. Desgleichen entfiel die Möglichkeit, die ohnehin bei den Massen unbeliebte Verbrauchersteuerd6) wesentlich zu erweitern. Der Krieg als Mittel zur Sanierung des Staatshaushaltes erschien als einziger Ausweg, um das revolutionäre System zu bewahren und das Vertrauen der finanzkräftigen Schichten wie das der einfachen Soldaten in die Liquidität der Regienung wiederherzustellen.

$\mathrm{Da}$ indes der Kriegsverlauf das Defizit anschwellen ließ und England auch nicht zur eindeutigen Suprematie über die Vereinigten Niederlande verhal ${ }^{47}$ ), schaltete Cromwell mit Hilfe des Heeres, das der Allianz zwischen Kaufleuten und Marine skeptisch gegenüberstand, die auf Fortsetzung des Krieges bis zur bedingungslosen Unterwerfung des Gegners drängenden Kräfte aus ${ }^{48)}$ und schloB unter einhelligem Protest von Kaufleuten, Handwerkern und religiösen Fanatikern Frieden. Das Ziel der protestantischen Vorherrschaft

Schach zu halten und damit eine direkte Konfrontation zu vermeiden, wurde im April 1652 vom Staatsrat abgelehnt.

44) Abbott 11 S. 535. Das Parlament erwog daher ernsthaft Pläne, Kathedralen wie die in Canterbury zu verkaufen bzw. abzureißen (Gardiner II S. 127).

45) Die èrste "General Assessment" zum Unterhalt der revolutionären Armee wurde am 24. Febr. 1643 vom Parlament erlassen. (Firth/Rait I S. 85 ff.). Am 19. Dez. 1651 wurde im Hinblick auf einen möglichen Krieg mit Holland eine „Assessment“ über monatlich 90000 Pfund auf sechs Monate beschlossen, die am 15. Juni 1652 um ein weiteres halbes Jahr verlängert wurde (Firth/Rait III S. LXXXVII und LXXXVIII) und am 10. Dez. 1652 auf 120000 Pfund monatlich ein halbes Jahr lang erhöht wurde (Firth/Rait II S. 653 ff.).

45) Die am 22. Juli 1643 eingeführte Akzise (Firth/Rait I S. 202 ff.) erfaBte ursprünglich nur alkoholische Getränke und Luxuswaren, wurde bald jedoch auf Lebensmittel und alltägliche Gebrauchwaren ausgedehnt. Diese Steuer, die während des Protektorats im Jahr etwa 400000 Pfund einbrachte, stieß auf heftigen Widerstand der lohnabhängigen Masse des Volkes. Ihre Abschaffung war ein Hauptanliegen der Levellers. Vgl. Anm. 26 und allgemein zur Akzise Ashley, Financial Policy S. 63 ff.

4) Ausführlich zum Kriegsverlauf Wilson S. 61 ff.

4) Am 20. April 1653 löste Cromwell das Rumpfparlament und den Staatsrat auf, die sich beide einem FriedensschluB widersetzt hatten. (Gardiner, History of the Commonwealth and Protectorate II S. 207 ff.). 
unter englischer Hegemonie verfolgte er wie bereits vor Ausbruch des Krieges jedoch weiter und bot den Niederlanden eine politische Union mit England und darüber hinaus eine gemeinsame Eroberung des spanisch-portugiesischen weltweiten Kolonialreiches $\left.a^{49}\right)$. Der Gedanke, England als Beschützer des protestantischen Interesses zur führenden Weltmacht aufsteigen zu sehen und in Abhängigkeit zu geraten, ließ die holländischen Unterhändler vor diesem Angebot zurückschrecken ${ }^{60}$ ). Erstmals hatte jedoch in der modernen Zeit ein puritanisch-calvinistisch geprägtes Staatengebilde, in der festen Uberzeugung, ein von Gott verliehenes überlegenes Wert- und Gesellschaftssystem zu verkörpern, seinen Anspruch auf eine internationale Führungsrolle geltend gemacht.

Der Mißerfolg des imperialistischen Handelskrieges mit Holland erschütterte das Vertrauen der religiös militanten unteren Mittelschichten $^{\text {(1) }}$ in die gottgewollte Führungsrolle Cromwells und entfremdete den Großteil der Kaufleute den demokratisch-revolutionären Ideen. Denn die Revolution schlug nach einem mißglückten als Mission verstandenen Krieg wieder in ihr Ursprungsland zurück und bedrohte dort in stärkerem Maße als zuvor die bestehende Eigentumsordnungsz). Der augenfällige Trend zur Restauration, zur Wiederherstellung einer dauerhaften staatlichen Ordnung, konnte auch durch Errichtung eines verfassungsmäßig abgesicherten Protektorats ${ }^{53}$ ) mit Cromwell in der halbmonarchischen Stellung eines Reichsverwcsers nicht aufgefangen werden. Allein auBenpolitische mit materiellem Gewinn verbundene Erfolge schienen in den

49) Ebenda S. 349 ff.: Angebot vom 23. Sept. 1653 über cinen Mittelsmann.

so) Ebenda S. 353 und S. 363: Im Oktober 1653 boten die Holländer den Abschluß eines Defensivbündnisses an. Der am 5. April 1654 in London unterzeichnete Frieden stellte im wesentlichen den status quo ante wieder her, schloß jedoch in einem Geheimabkommen die mit den Stuarts verwandten Oranier von der holländischen Statthalterschaft aus (Ebenda $S$. 370 ff.).

51) Das „Barebones Parlament“ (das „Parlament der Heiligen“), das vom 4. Juli bis 12. Dezember 1653 tagte, war das politische Spradirohr dieser Schichten. Da es auf Abschaffung des Zehnten und eine Justizreform drängte, wurde es von Cromwell zur Selbstauflösung gezwungen. Ausführlich bei $A$ bbott III S. 48 ff.

s2) Brenner S. $105 \mathrm{f}$. und Farnell S. 452: Die traditionelle Kaufmannschaft gewann mit der Auflösung des „Barebones“-Parlament ihren beherrschenden Einflub zurïck.

53) Gardiner, Constitutional Documents S. $314 \mathrm{ff}$. und Firth/Rait Il S. 813 ff.: Instrument of Government vom 16. Dez. 1653. 
Augen des stärker pragmatisch orientierten neuen Staatsrates dazu angetan, das Volk hinter der neuen Regierung zu einigen.

Hatten die englischen Kräfte für eine Auseinandersetzung mit cinem gleichwertigen Gegner nicht ausgereicht, so bot der seit 1635 anhaltende spanisch-französische Krieg Koalitionsmöglichkeiten und darüber hinaus die Chance eines profitablen Raubzuges gegen „undurchkapitalisierte Territorien “54). Noch vor der förmlichen Unterzeichnung des Friedensvertrages mit Holland kreiste daher die Debatte im Staatsrat ${ }^{55}$ ) um die Frage, wie die starke englische Marine und das politisch unzuverlässige Heer möglichst gemeinsam entweder gegen Spanien oder Frankreich eingesetzt werden könnten. Ein englisches Stillhalten, so hieß es unumwunden, sei nur denkbar, wenn beide Seiten dafür bezahlten, und ein Krieg gegen die spanischen Besitzungen in Westindien vermutlich doch am lukrativsten. Obgleich Frankreich sich weigerte, die englische Freundschaft zu kaufen - sollte es doch die zur Eroberung Dünkirchens als britischem Handels- und Marinestützpunkt vorgesehenen englischen Truppen bezahlen ${ }^{56}$ - gewann der Plan eines auf die Karibische See zu lokalisierenden Kolonialkrieges im Verlauf des Jahres 1654 die Oberhand ${ }^{57}$ ).

Außenwirtschaftliche Gründe, von der alten Anti-HollandLobby erneut vorgebrachts8), sowie damit aufs engste verknüpfte Fragen eines stabilen Währungssystems sowie letztlich wiederum in der Person Cromwells verkörperte Gedanken eines protestantischen Weltreiches beeinflußten diese Entscheidung mit. Bereits unter den Stuarts hatte während des Seekrieges mit Spanien (1625-29) die

54) $\mathrm{Zu}$ diesen beiden Formen kapitalistischer Expansionspolitik siehe Hallgarten I S. 30.

s) Abbott III S. 260 f.: Sitzung vom 20. April 1654.

50) Für das „Feilschen“ Cromwells mit beiden Seiten siche Gardiner, History of the Commonwealth and Protectorate II S. $421 \mathrm{ff}$. Dabei sprachen englische Diplomaten offen vor dem französischen Botschafter aus, daß Cromwell im englischen Staatsinteresse die Truppen beschäftigen miisse, dafür jedoch ausländische Subventionen benötige (Ebenda S. 468).

57) Ebenda S. $474 \mathrm{ff}$.: Nachdem Spanien im Krieg mit Frankreich am 24. Aug. 1654 Arras verloren hatte, wurde das Projekt eines englischen Vorstoßes nach Westindien ernsthaft betrieben.

58) Farnell S. 453, Prestwich S. 108, Ashley, Financial Policy S. 133 ff.: Interlopers um Lawrence Thompson, der Plantagen auf Barbados besa $\beta$ und Tabak in Virginia aufkaufte, sowie die Gebrüder Noell, Privatfinanziers von Cromwell und Lieferanten der Flotte, unterstützten im Gegensatz zur traditionellen Kaufmannschaft den westindischen Kriegsplan. 
Kombination von elisabethanischem Freibeutertum und Kolonisationsinteressen einer kleinen Gruppe zum Puritanismus tendierender meist adliger Großgrundbesitzer ${ }^{59}$ ) und freier Londoner Kaufleute ${ }^{60}$ ) zur Annexion der spanischen Antilleninsel Barbados geführt. Das feudale spanische Kolonialprinzip wurde durch das kapitalistische englische ${ }^{61}$ ) der rationalen, monokulturellen Plantagenwirtschaft herausgefordert. Da die englische Pflanzeraristokratie allerdings auf Arbeitssklaven ebensowenig verzichten konnte wie dic Herren auf den Haciendas, bildete sich in den englischen Kolonien Amerikas in den 30er und 40er Jahren des 17. Jahrhunderts eine unterschiedliche Sozialordnung heraus. Dieser Unterschied zwischen dem selbständigen, in die puritanische Religionsgemeinschaft eingebetteten Grenzfarmer des Nordens und dem profitorientierten Großgrundbesitzer des Südens zeitigte erstmals nach dem englischen Bürgerkrieg politische Folgen, als sich die südlichen Kolonien weigerten, die Commonwealth-Regierung anzuerkennen, und ihre Plantagenprodukte selbständig mit Hilfe holländischer Frachtschiffe exportierten ${ }^{82}$ ). Da sich der Handel mit Tabak und Zucker als genauso gewinnbringend wie der Import indischen Pfeffers ${ }^{88}$ ) erwies, dabei aber das unternehmerische Risiko des langen, gefahrvollen Seeweges entfiel, veranlaßten independentische City-Kreise die Verhängung eines Handelsembargos über die Kolonien und drangen auf eine gewaltsame Durchsetzung der Navigationsakte im westlichen Atlantik, um den verlorenen Handel wicder unter Kontrolle zu bekommen ${ }^{84}$ ). Die Commonwealth-Regierung ihrerseits benutzte die Handelsge-

59) Stone S. $364 \mathrm{ff}$.

00) Diese Interessenkoalition war in der "Providence Island Company" manifest, die 1636 private Raubzüge in die Karibische See unternommen hatte (Hill, Century of Revolution S. 40). Zum Kriegsausbruch mit Spanien 1624: G. E. Aylmer: The Struggle for the Constitution. England in the Seventeenth Century. London 1963. S. $64 \mathrm{ff}$.

(1) Zu dieser Abgrenzung Max Weber: Wirtschaftsgeschichte. Abriß der universalen Sozial- und Wirtschaftsgeschichte. Berlin ${ }^{3} 1958$. S. 255.

a2) Gardiner, History of the Commonwealth and Protectorate II S. 75: Barbados, Bermuda, Maryland und Virginia blieben royalistisch, während die Sympathien der Siedler Neuenglands und Neufundlands auf seiten des Parlaments lagen.

63) Ein Pfund Pfeffer wurde in Indien mit $2 \mathrm{~d}$ gekauft und in London mit $20 \mathrm{~d}$ verkauft. Zu den Gründerjahren der Ostindienkompanie siehe $K$. $N$. Chaudhuri: The English East India Company. A Study of an Early JointStock Company 1600-1640. London 1965.

64) Vgl. Anm. 58. 
setze als Mittel politischer Disziplinicrung, konnte sich aber während des Krieges mit Holland nicht voll durchsetzen ${ }^{65}$ ). Ein Vordringen in das spanisch beherrschte Westindien mit dem Ziel, die reichste und am weitesten kultivierte Insel Hispaniola (heute: Haiti, Dominikanische Republik) zu annektieren, mußte zwangsläufig die Kolonien wieder stärker an das Mutterland binden und den beiden Maximen Cromwellscher und späterer englischer Außenpolitik, „state power“ und "merchant profit“, genügen.

Uberdies versprach eine Besetzung Hispaniolas die spanischen Silberimporte aus Südamerika kontrollieren zu können, die das europäische Währungssystem nun beinah schon seit hundert Jahren aushöhlten. Die in England seit etwa Ende des 16. Jahrhunderts rapide ansteigenden Lebenshaltungskosten bei stagnicrenden bzw. in lohnabhängigen Arbeiterkreisen eher sinkenden Realeinkommen ${ }^{86}$ ) ein Pfund Butter kostetc um 1650 den Tagesverdienst eines Arbeiters $\left.^{67}\right)$ - wurden einhellig auf das unkontrolliert in die nationalen Märkte einfließende spanische Edelmetall zurückgeführt ${ }^{\text {08) }}$. Doch

a5) Gardiner, History of the Commonwealth and Protectorate II S. $75 \mathrm{ft.}$ 1652 hatte ein nach Amerika entsandter kleiner Flottenverband nur einen Kompromiß erzwingen können. Die ehemals royalistischen Kolonien erkannten die politische Oberhoheit der neuen Londoner Regierung an, bewahrten sich jedoch finanzielle und kommerzielle Unabhängigkeit.

o6) Zum Preisauftrieb: Y. S. Brenner: The Inflation of Prices in England 1551-1650. In: „The Economic History Review“, 1962, S. 266-284. Demnach stieg der Lebensmittelindex von 1551100 auf 229 im Jahrzehnt 1641-1650.

Zu den Löhnen: Ebenda und Kuczynski S. 149; zu den Einkommen der Selbständigen: Wolfe S. 311 ff., Stone S. 762 (demnach lag das Durchschnittseinkommen der Peers 1641 bei 6030 Pfund, nach dem Preisindex von 1559 2740 Pfund). Genaue Zahlen für 1600 und 1688 bei Cooper/Thirsk S. $751 \mathrm{ff}$. und S. $780 \mathrm{f}$. Das Durchschnittseinkommen der Peers war ca. 600mal höher als das der Arbeiter, denen Tageslöhne von minimal (auf dem Lande) $4 \mathrm{~d}$ bis maximal $15 \mathrm{~d}$ gezahlt wurden.

o7) Wolfe S. 311 und Cooper/Thirsk S. $48 \mathrm{ff}$. (aus dem Tagebuch eines Landpfarrers in Essex 1646-1651)

98) Als erster hatte Bodin den Preisauftrieb in Europa mit den spanischen Sitberimporten erklärt $(M$. E. Kamp: Die Staatswirtschaftslehre Jean Bodins. Bonn 1949). Zu den englischen Theoretikern des 17. Jahrhunderts siehe: Jürgen Kuczynski: Zur politökonomischen Ideologie in England und andere Studien. Berlin (Ost) 1965; Rudolf Hilferding: Aus der Frühzeit der englischen Nationalökonomie. In: „Die Neue Zeit“", 29, 1910 S. 908 ff. (im wesentlichen eine Auseinandersetzung mit der Schrift von Thomas Mun: England's Treasure by Foreign Trade, 1664; jetzt auffindbar bei $J$. $R$. McCulloch (Hrsg.) Early English Tracts on Commerce. Cambridge 1952. 
über die Lösung der Währungsfrage differierten die stets von der eigenen Interessenlage beeinflußten Ansichten der Kaufleute von ciner Zwangsdevisenbewirtschaftung mit festen Wechselparitäten ${ }^{\text {BD) }}$ bis hin zu den Vorstellungen einer aktiven Außenhandelsbilanz, die auch, so die Verteidiger der „East India Company ${ }^{400}$ ), durch den eigentlich verbotenen Kapitalexport zu erreichen sei. Dirigistische Eingriffe des Staates in die Wirtschaft, die frühmerkantilistischen Schutzmaßnahmen der handeltreibenden curopäischen Länder, entsprangen daher weniger absolutistischer Fürstenwillkür, sondern wurden durch die bankrotten Staatsfinanzen des Silberexporteurs Spanien ${ }^{71}$ ) diktiert und galten gerade in England unter den frühen Stuarts der Sorge um das Wohl der Iohnabhängigen Untertanen. Zwar fühlte sich die Regierung Cromwell derartiger Fürsorgepflichten cnthoben ${ }^{72}$ ), dafür griffen aber die am Westindienhandel interessierten Kaufmannskrcise zusammen mit Finanziers die Idee auf, den seit 1629, dem letzten FriedensschluB mit Spanien, erzwungenen Silberabflu (7a) $^{73}$ die englische Münze zu vergrößern und damit die englische Währung zu sanieren.

Doch abgesehen von dieser kleinen Gruppe opponierte die traditionelle Londoner Kaufmannschaft, die inzwischen wieder die politische Macht in der City besaß, gegen einen Krieg mit dem spanischen Haupthandelspartner und verweigerte Cromwell bis zu seinem

S. $115 \mathrm{ff}$.); ferner die Dissertation von Hjalmar Schacht: Der theoretische Gehalt des englischen Merkantilismus. Berlin 1900; am ausführlichsten Schulin S. $61 \mathrm{ff}$. und die Darstellung von Ernst Klein: Die englischen Wirtschaftstheoretiker des 17. Jahrhunderts. Darmstadt 1973.

60) Vorwiegend von Gerard de Maynes (Consuetudo vel Lex Mercatoria, 1622) vertreten. Siehe Schulin S. 61 f. und Klein S. 22 ff.

70) Besonders Thomas Mun, einer der Direktoren der „East India Company". Dazu McCulloch S. 115 ff. und Schulin S. 74 ff. sowie Klein S. 40 ff. 71) 1557, 1575, 1596, 1607 mußte die spanische Regierung den Staatsbankrott anmelden; der Preisanstieg betrug im 16. Jh. in Spanien 400\%. Dazu mit weiterführender Literatur: Hans Rabe: Die iberischen Staaten im 16. und 17. Jahrhundert. In: „Handbuch der Europäischen Geschichte“, hrsg. von Theodor Schieder, Bd. III, Stuttgart 1971, S. 631 f. and 641 f. sowie ebenda S. 298 f.

78) Zur auf allen Sektoren mangelhaften Sozialpolitik des englischen Parlaments, das zwischen 1640 und 1660 kein einziges Gesetz zur materiellen Besserstellung der Arbeiter und auch keine Maßnahmen in der Armengesetzgebung traf, die ausgezeichnete Arbeit von James.

73) Y. S. Brenner, Infation of Prices S. $277 \mathrm{ff}$. und Supple S. 165. Die Verrechnung dieses Silbers, aus dem die englische Münze zwischen 1631 und 16437 Millionen Pfund prägte, erfolgte über Amsterdam. 
Tode jede Anleihe74). Der drohende Staatsbankrott - 1654 verschlang trotz höchster Besteuerungsrate während der Protektoratszeit allein das Armeebudget die gesamten Staatseinnahmen ${ }^{75}$ ) - und somit das abzusehende Ende seiner Herrschaft zwangen Cromwell in viel stärkerem Maße als im Konflikt mit Holland zur Flucht nach vorn, zu einem hastigen, ungenügend geplanten Raubzug nach den spanischen Silberschätzen ${ }^{76}$ ), um die aus dem Lande selbst nicht mehr zu bezahlenden Streitkräfte als unabdingbaren Schutz seiner Regierung zu unterhalten. Die religiöse Verbrämung dieser Aggression als eines Kreuzzuges gegen das papistische Spanien, das die Eingeborenen mißhandele und ausrotte ${ }^{77}$ ), gewann die alteingesessene, elisabethanische "Gentry“ und die städtischen Massen vorübergehend für den $\mathrm{Krieg}^{7 \mathrm{f}}$ ), beinhaltete jedoch auch erncut den protestantischen Führungsanspruch des englischen Volkes und übertrug ihn erstmals, als Mission verstanden, in kolonjale, in ihrer wirtschaftlichen Entwicklung zurückgebliebene Gebiete. Die englisch-puritanische Sozialordnung, eine Mischung aus nationalem Auserwähltheitsglauben und rationalem Wirtschaftsimperialismus, wurde damit erstmals unter Cromwell zu cinem Exportartikel, zu eincr außenpolitisch tragfähigen Ideologie erhoben. Der Fehlschlag des Missionszuges79) - nur durch Zufall wurde nach einem Desaster auf Hispaniola schließlich

74) Ashley, Financial and Commercial Policy S. 99 und Prestwich S. 116.

73) Ashley, Financial and Commercial Policy S. 48 und 96: Die Staatseinkünfte beliefen sich auf 1.586 Mill. Pfund, von denen der Armeehaushalt 1.566 umfaBte. Firth/Rait II S. 902 ff. Durch eine Ordonnanz Cromwells vorn 8. Juni 1654 wurde die monatliche „Assessment"-Rate auf 120000 Pfund angehoben. Vgl. Anm. 45.

76) Selbst Gardiner, History of the Commonwealth and Protectorate III S. 342 ff. kritisiert die übereilten militärischen, noch dazu ungenügenden Vorbereitungen.

In den militärischen Instruktionen Cromwells stand der Befehl: „Be master of the Spanyards Treasure" (Abbott III S. 536, Anweisung an General Venables vom 9. Dez. 1654).

71) Abbott III S. 533 Anweisung an General Venables vom 4. Dez. 1654: .... (Spain) hath not only exercised inhuman cruelties upon the natives, and prohibited all other nations to have any trade ... but hath ... expelled the pcople of these islands ... destroying and murdering many of their men ..."

78) Das am 17. Sept. 1656 einberufene Parlament, vor dem Cromwell in seiner Eröffnungsrede Spanien als "natural enemy" bezeichnete ( $A$ bbott IV S. 260 ff.), billigte den Krieg.

7") Auf Hispaniola schlugen etwa 200 spanische Viehhirten 9000 "godly soldiers" in die Flucht. Zum Kriegsverlauf: Gardiner, History of the Commonwealth and Protectorate III S. 355 ff. 
Jamaica okkupiert - besiegelte den allmählichen Staatsbankrott ${ }^{80}$ ). Dies wiederum beschleunigte den seit 1653 kontinuierlichen Autoritätsschwund des Cromwellschen Regierungssystems und dürfte zusammen mit dem Tode des Protektors der Hauptgrund für die Rückkehr des Königs gewesen sein. Aus mangelnder innerer Stabilität hatte sich das revolutionäre England in zwei Kriegen, gegen einen ebenbürtigen und einen kolonialen Gegner übernommen, mit diesen Unternehmen indes Grundlinien für das spätere immer auf diesen beiden Wegen - Ausschalten eines übermächtigen Konkurrenten und Durchdringen unterentwickelter Kolonialräume - vorwärtsgetriebene imperiale Ausgreifen gesetzt und dabei bereits unter Cromwell mehr aus Zwang denn aus strategischem Kalkül den Grundsatz des politischen Gleichgewichts entdeckt und im Ostseeraum ${ }^{81}$ ) praktiziert.

Eine „Balance of Power"-Strategie im nördlichen Europa wurde England damals durch seine Importabhängigkeit, vornehmlich im Hinblick auf Schiffsbaumaterialien, diktiert. Getreide- und Erzeinfuhren aus den Ostseeländern waren im 17. Jahrhundert noch von untergeordneter Bedeutunga2). Während des Krieges mit Holland hatte die Sperre des Sundes durch die Dänen ${ }^{85}$ ) die Seemacht England erstmals mit einer Meerengenfrage als lebenswichtigem Politikum konfrontiert. Cromwell sicherte sich nach dem Frieden mit den Niederlanden sofort durch Handelsverträge mit Dänemark und Schweden ${ }^{84}$ ) ab und errcichte schließlich durch diplomatisches Taktie-

80) Als Cromwell starb, betrugen die rückständigen Soldzahlungen allein für die Armec 1,2 Mill. Pfund. Sein Sohn und Nachfolger im Amt des Protektors Richard C. konnte wegen der desolaten Staatsfinanzen nicht einmal das Begräbnis bezahlen und ersuchte deswegen die französische Regierung um eine Anleihe von 50000 Pfund (Ashley S. $105 \mathrm{ff}$., der trotz aller erarbeiteten Fakten über den finanziellen Zusammenbruch des Protektorats resümierend konstatiert: „It is therefore quite clear that it was Richard's weakness not Oliver's debts that overthrew the Cromwellian Protectorate").

81) Ausführlich zu Cromwells Ostseepolitik: Michael Roberts: Cromwell and the Baltic. In: „The English Historical Review“, 1961, S. 402-446.

82) Vgl. Anm. 33.

88) Gardiner, History of the Commonwealth and Protectorate II S. 82, 140 und 154 f. Nachdem der dänische König (Friedrich III) im Sept. 1652 bereits 20 englische Schiffe mit Schiffbaumaterialien im Sund aufgehalten hatte, verpflichtete er sich in einem Vertrag mit Holland vom 29. Jan, 1653, während des englisch-holländischen Krieges keine englischen Schiffe passieren zu Jassen.

84) Vertrag mit Schweden vom 11. April 1654 (Wortlaut bei Abbott III S. 911 ff.). Vertrag mit Dänemark vom 15. Sept. 1654, in dem Eng- 
ren im schwedisch-dänischen Krieg, daß beide als Anrainer des Sundes ab 1658 diese Schiffahrtspassage gemeinsam kontrollierten ${ }^{85}$ ). Das wirtschaftliche Erfordernis eines ungehinderten englischen Handels in den europäischen Binnenmeeren lieB Cromwell zur selben Zeit auch den Gedanken erwägen, Gibraltar als Stützpunkt zu besetzen $^{86)}$.

Selbst die Idee eines Kondominiums mit der wenn damals noch nicht stärksten so doch flächenmäßig größten europäischen Landmacht, eines Europa überspannenden und kontrollierenden englisch-russischen Flügelbündnisses ${ }^{87}$ ), läßt sich in Ansätzen bereits in der englischen Revolution fassen. Um die 1613 nach einer nationalen Erhebung zur Macht gelangte neue Dynastie der Romanovs gegen die von Schweden, Polen und Habsburg ausgehende Bedrohung außenpolitisch abzusichern ${ }^{88}$ ), hatte eine russische Gesandtschaft 1645 in London ein Buindnisangebot unterbreitet ${ }^{80}$ ). Die zur Bedingung erhobene Wiederherstellung aller monarchischen Praerogativen hätte in der Situation des Bürgerkrieges die Kapitulation des Parlaments bedeutet und wurde daher verworfen. Nach der Hinrichtung Karls I. übte allein der russische Zar monarchische Solidarität. Er ließ den englischen Besitz konfiszieren und die englischen Kaufleute, die Träger der antiroyalistischen Revolution, in seinem Machtbereich verhaften ${ }^{80}$ ). Vergeblich bemïhte sich Cromwell in der Protektoratszeit, das russische Angebot wieder aufzugreifen. Vermittlungsbestre-

land hinsichtlich der pauschalen Begleichung der Sundzölle Gleichrangigkeit mit den Vereinigten Niederlanden erreichte (Wortlaut bei Abbott III S. $925 \mathrm{ff}$.

ss) Roberts S. 436 und Prestwich S. 114: Frieden zu Roskilde vom 26. Febr. 1658, in dem Dänemark die Gebiete östlich des Sunds, Schonen und Blekinge, an Schweden abtrat.

8) Abbott IV S. 641 und Ramsay S. 58.

87) Uber die frühen Beziehungen zwischen England und RuBland: $J$. Lubimenko: Les relations commerciales et politiques de l'Angleterre avec 1a Russie avant Pierre le Grand. Paris 1933; speziell zur Frühgeschichte der 1553 gegründeten ältesten engliscten Handelskompanie, der „Muscovy Company", siehe T. S. Willan: Trade between England and Russia. In: "The English Historical Review", 1948, S. 307-321; ferner zur Protektoratszeit: Z. I. Roginskij: Iz istorii Anglo-Russkich otnošenij v period Protektorata Kromvelja. In: „Novaja i novejకaja istorija“, Moskva, 1958, S. 71-78.

89) Dazu allgemein: Günther Stökl: Russische Geschichte von den Anfängen bis zur Gegenwart Stuttgart 1965. S. 276 ff.

80) Ashley, Commercial and Financial Policy, S. 118.

\$o) Firth, Oliver Cromwell S. 233. 
bungen im schwedisch-russischen-polnischen Konflikt in der Absicht, eine antikatholische Koalition zwischen Protestantismus und russischer Orthodoxic zu begründen, konnten ebenso wenig wie verlockende Handelsangebote und die englische Unterstïtzung des antipolnischen auf Vereinigung mit Rußland abzielenden Aufstandes der Kosaken den Protektor in den Augen des Zarenhofes vom Makel des Usurpators befreien"1). Wohl zumindest in partieller Reaktion auf den religiös-sozialen Wandel in Europa vollzog sich so unbeschadet dirckter ausländischer Einflußnahme die Konsolidierung der zaristischen Selbstherrschaft, während zur gleichen Zeit England unter Cromwell den Weg zu einer parlamentarischen Regienungsform beschritt.

Wie Cromwell weder als Initiator noch als treibende Kraft der revolutionären Umgestaltung, sondern eher als um Vermittlung bemühter Vollstrecker divergierender Interessen inmitten einer wirtschaftlichen Zwangslage gesehen werden sollte, so kann diese Epoche in der englischen Geschichte auch nicht als die entscheidende Zäsur, sondern besser als Katalysator und zugleich Scheitelpunkt eines langwierigen ökonomischen, sozialen und politischen Umwandlungsprozesses gedeutet werden. Zwar kommt dem Staatsbankrott für die Außenpolitik und - nach deren Fehlschlag - für die unaufhaltsame Vertrauenseinbuße der Regierung Cromwell und somit letztlich für das Scheitern der Revolution entscheidende Bedeutung zu. Eine rein ökonomistisch-klassenspezifische Interpretation verbietet sich aber wiederum angesichts des politischen Eigengewichts, das Cromwells "1) Auf Anregung der „Muscovy Company“ beschloß der "Council of State“ im Juni 1654, einen Gesandten an den Zarenhof zu senden, um die Wiederaufnahme des Handels zu erreichen und dic russischen Motive für den Kricg mit Polen zu erfragen (Abbott III S. 341 u. 622). Polen, seit dem Aufstand der Zaporoger Kosaken (1654) im Krieg mit Rußland, hatte über cinen Sondergesandten im März 1654 vergeblich um englische Flottenunterstützung, einen Vorstoß gegen Archangelsk, nachgesucht (Ebendit S. 560). Obwohl der englische Gesandte Moskau erreichte und einen nicht. überlieferten Brief Cromwells dem Zaren überreichte, erwartete Aleksej Michailovič die Wiederherstellung der Monarchie in England und weigerte sich daher, mit der Protektoratsregierung Kontakt aufzunehmen (Ebenda S. 754). Eine zweite Mission (1657) mit dem Ziel, zwischen Schiweden und RuBland zu vermitteln (Ebenda Bd. IV, S. 449 ff.: Schreiben Cromwells an den Zaren vom 7. 4. 1657 und die ausführlichen Instruktionen an den Gesandten), scheiterte bereits bei der Einreise, da die Russen die Titulator des Zaren in Cromwells Schreiben für ungenügend erachteten (Ebenda $S$. 572), in Wirklichkeit jedoch an einer englischen Vermittlung desinteressiert waren. $\mathrm{Zu}$ den englischen Kontakten mit den Kosaken siehe Roginskij. 
"einsamen Entschlüssen“ - seiner Opposition gegen den Volkskrieg mit Holland sowie seinem starrköpfigen Beharren auf dem westindischen Kriegsplan - beizumessen ist.

Während der Herrschaft Cromwells brechen allerdings erstmals gesellschaftliche, wirtschaftliche und machtpolitische Maximen durch, die jedoch, um Englands weitere Entwicklung zur vorherrschenden See- und Handelsmacht zu bestimmen, der Legalisierung durch die Machtübernahme der besitzenden Oberschicht in der "glorious revo-

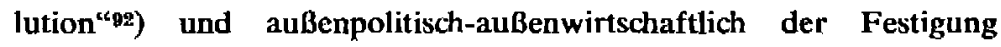
durch den Spanischen Erbfolgekrieg bedurften ${ }^{\text {Da }}$ ). Der von Cromwell aus finanzieller Notlage wic auf den Druck bestimmter wirtschaftlicher Interessengruppen hin organisicrte Außenhandelskapitalismus, die Ubertragung des staatlichen Schutzes von den Handelskompanien auf den gesamten nationalen AuBenhandel, und die Durchsetzung dieses Prinzips in imperialistischen Handelskriegen mit dem innenpolitischen Ziel, das Regicrungssystem zu festigen - diesc Politik wurde von den zurückgekehrten Stuarts sogleich im Interesse des Staates weitergeführt ${ }^{\text {94)}}$. Hingegen kappte die Restauration 1660 die mit dem monarchischen Prinzip nicht zu vercinbarende puritanische Ideologic der göttlichen Auserwähltheit des gesamten englischen Volkes ${ }^{95}$ ).

a2) Mit der auf die „Glorreiche Revolution“ (1688) folgenden „Declaration of Rights" (1689) wurde die parlamentarisch-konstitutionelle Monarchie in England begründet. Die in der Deklaration enthaltene Bestimmung, kein stehendes Heer in England zu unterhalten, entschied den seit dem Bürgerkrieg anhaltenden Streitfall zwischen der Zentralgewalt und der Gentry bzw. den Kaufleuten über die Stellung der Armee, an der das Protcktorat zerbrochen war, im Sinne der besitzorientierten Oberschicht.

na) Im Separatfrieden zu Assiento im März 1713 sicherte sich England auf Druck der am Uberscehandel interessierten Kreise das Monopol des Sklavenhandels mit den spanischen Kolonien in Amerika; im Frieden zu Utrecht baute es dann seine koloniale Position in Nordamerika auf Kosten Frankreichs aus und übernahm Gibraltar von Spanien.

94) Die Navigationsakte wurde in verbesserter Form sogleich von Karl II. 1660 erneuert (Cooper/Thirsk S. 520 ff. Wortlaut). Der Kommentar des Sprechers im Unterhaus zu diesem Vorgang: „Dies ist der einzige Wcg, um Ew. Majestät Herrschaft über die ganze Welt auszubreiten" (Bentin S. 49), gibt wohl treffend die volle Zustimmung des Parlaments mit einer expansiven, am Außenhandel orientierten Weltmachtpolitik Englands wieder.

85) Unter der verschärften staatskirchlichen Gesetzgebung der Restauration (Clarendon-Code) waren etwa ein Drittel der Bevölkerung „Dissenters“, so dab analog zu den dreißiger Jahren in den 60er und 70er Jahren des 17. Jh. eine Auswanderungswelle religiös verfolgter Puritaner nach Nordamerika zu verzeichnen war. 
Dieses Gedankengut sollte in den puritanischen Siedlerkolonien Neuenglands fortleben und in säkularisierter Form in das demokratische Sendungsbewußtsein des amerikanischen Volkes einfließen. Die USA sollten schließlich England in der angelsächsischen Führungsrolle ablösen und dabei in modifizierter Form die in der Zeit Cromwells begriindeten englischen außenpolitischen Leitgedanken iubernehmen, wie den des Aquilibriums und der Sicherung der Gegenkiuste als Voraussetzungen eines ebenfalls maritim abgesicherten imperialistischen Hegemonialstrebens ${ }^{90}$ ).

\$) Dazu, jedoch stärker unter geopolitischen Gesichtspunkten, auch: Ludwig Dehio: Gleichgewicht oder Hegemonie. Betrachtungen über ein Grundproblem der neueren Staatengeschichte. Krefeld 1948. 